\title{
Transarterial Chemoembolization Combined with Sorafenib in Patients with BCLC Stage C Hepatocellular Carcinoma
}

This article was published in the following Dove Press journal: Drug Design, Development and Therapy

\author{
Kai-Cai Liu (D) ${ }^{1, *}$ \\ Ying-Hong $\mathrm{HaO}^{2}$ ** \\ Wei-Fu Lv ${ }^{2}$ \\ Wei-Dong Jia ${ }^{3}$ \\ Chu-Shu ji ${ }^{4}$ \\ Chun-Ze Zhou ${ }^{2}$ \\ De-Lei Cheng $\left(\mathbb{D}^{2}\right.$ \\ Shao-Bao $\mathrm{Xu}^{\prime}$ \\ Zong-Gen Gao' \\ Ming-Xue Su' \\ Chang-Sheng Shi'
}

'Infection Hospital, Anhui Provincial Hospital, The First Affiliated Hospital of USTC, Division of Life Sciences and Medicine, University of Science and Technology of China, Hefei 230000, People's Republic of China; ${ }^{2}$ Department of Radiology, The First Affiliated Hospital of USTC, Anhui Provincial Hospital, Division of Life Sciences and Medicine, University of Science and Technology of China, Hefei, Anhui 230022, People's Republic of China; ${ }^{3}$ Department of General Surgery, The First Affiliated Hospital of USTC, Anhui Provincial Hospital, Division of Life Sciences and Medicine, University of Science and Technology of China, Hefei, Anhui 230022, People's Republic of China; ${ }^{4}$ Medical Oncology, The First Affiliated Hospital of USTC, Anhui Provincial Hospital, Division of Life Sciences and Medicine, University of Science and Technology of China, Hefei, Anhui 230022, People's Republic of China

*These authors contributed equally to this work

Correspondence: Wei-Fu Lv Department of Radiology, The First Affiliated Hospital of USTC, Anhui Provincial Hospital, Division of Life Sciences and Medicine, The First Affiliated Hospital of USTC, Division of Life Sciences and Medicine, University of Science and Technology of China, Hefei, Anhui 230022, People's Republic of China $\mathrm{Tel} / \mathrm{Fax}+8655162283139$

Email weifulv@ustc.edu.cn
Purpose: Transcatheter arterial chemoembolization (TACE) and targeted therapy have become common methods in the treatment of advanced hepatocellular carcinoma (HCC). The purpose of this study was to evaluate the safety and efficacy of TACE combined with sorafenib (TACEsorafenib) and TACE alone for the treatment of Barcelona clinical stage C HCC.

Methods: The clinical data of 75 patients with BCLC stage C HCC who received TACEsorafenib or TACE as the initial treatment were retrospectively analyzed. Tumor response, time to progression (TTP), overall survival (OS), and adverse events were compared at 1 month after surgery in the two groups.

Results: One month after treatment, the disease control rate in the TACE-sorafenib group was higher than that in the TACE group alone $(82.76 \%$ and $57.50 \%$, respectively, $\mathrm{P}=0.018)$. The median values of TTP and OS in the TACE-sorafenib group were longer than those in the TACE group (TTP was 7.6 and 3.4 months, respectively, $\mathrm{P}=0.002$; OS was 13.6 and 6.3 months, respectively, $\mathrm{P}=0.041$ ). The cumulative survival time at 3 months, 6 months, and 1 year was higher in the TACE-sorafenib group than in the TACE group $(83.5 \%, 71.2 \%, 45.7 \%$ vs $57.4 \%$, $40.6 \%, 21.2 \%$ ). Sorafenib-related side effects such as hypertension, hand-foot syndrome, and oral ulcers were more common than those in the TACE group alone $(\mathrm{P}<0.05)$.

Conclusion: Compared with TACE treatment alone, TACE combined with sorafenib in BCLC-C stage HCC significantly improved disease control rate, TTP, and OS, and no significant increase in adverse reactions was observed.

Keywords: hepatocellular carcinoma, TACE, sorafenib, survival

\section{Introduction}

Hepatocellular carcinoma (HCC) is one of the common malignant tumors with high mortality in the digestive system. ${ }^{1}$ It has the characteristics of occult disease, high malignancy, fast growth, wide metastasis, short natural course and high recurrence rate. ${ }^{2,3}$ Surgical resection is the first choice for the treatment of early HCC, but most patients have advanced cancer when diagnosed, and the effect of resection is poor. ${ }^{4}$ Meanwhile, the sensitivity of HCC to the radiotherapy and chemotherapy is also unsatisfactory. ${ }^{5}$ Transcatheter arterial chemoembolization (TACE) is mostly used in patients with advanced HCC who do not meet the condition of radical resection. ${ }^{6,7}$ TACE alone is often difficult to obtain a satisfactory curative effect, especially in the patients with advanced HCC. The reasons are partially contributed to the increase of vascular endothelial growth factor induced by the heightening of hypoxia after tumor necrosis, thereby increasing the formation of tumor 
vessels. ${ }^{8,9}$ Therefore, it is essential to give an antiangiogenesis for patients who accepted TACE treatment for improving clinical efficacy, reducing tumor recurrence and metastasis, and prolonging the survival. Sorafenib is an oral multikinase inhibitor, which can directly inhibit the Ras/Raf/MEKUERK signaling pathway to inhibit the proliferation of tumor cells, and can also inhibit the activity of related receptors related to neovascularization. It can inhibit the secretion of VEGF and other cellular inflammatory factors, thereby blocking the formation of tumor new blood vessels and inhibiting the growth of tumors. According to the criteria for the diagnosis and treatment of Barcelona Clinic Liver Cancer system, patients with HCC in BCLC stage B (Child-Pugh grade A to B) are preferred for TACE therapy, and patients in stage $\mathrm{C}$ are treated with sorafenib. ${ }^{10}$ While if TACE in combine with sorafenib can obtain an improved effect for patients with BCLC stage C HCC is uncertain. This study conducts a retrospective analysis to evaluate the effect of TACE combined with sorafenib.

\section{Materials and Methods}

This study was carried out in accordance with the principles set out in the declaration of Helsinki in 1964. Approved by the ethics committee of the First Affiliated Hospital of the University of Science and Technology of China, through the retrospective study design and analysis of clinical data, the ethics committee of the First Affiliated Hospital of the University of Science and Technology of China formally gave up informed consent. All patient information is confidential.

\section{Study Population}

A total of 307 patients with stage BCLC-C HCC treated in the First Affiliated Hospital of the University of Science and Technology of China from January 2010 to December 2018 were collected. The inclusion criteria were as follows: (1)the diagnostic standards being in line with the criteria endorsed by the American Association for the Study of Liver Diseases; ${ }^{2}$ (2)stage C HCC based on BCLC staging criteria, ${ }^{11}$ (3)Childpugh classification of A or B; (4)Eastern Cooperative Oncology Group (ECOG) performance status (PS) of 0 or 1; (5)white blood cell count $\geq 2000 / \mu \mathrm{L}$, hemoglobin $\geq 8.0 \mathrm{~g} / \mathrm{dL}$, and platelet count $\geq 6.0 \times 10^{4} / \mu \mathrm{L}$; (6)tumors size less than $70 \%$ of the whole liver volume; Exclusion criteria included: patients who had undergone systemic chemotherapy, targeted therapy or radiofrequency ablation (RFA) previously; (2)patients with other primary malignancies, or other concurrent serious medical conditions;(3)patients who had contraindications to TACE treatment; (4)severe coagulopathy; This study finally included 84 patients with stage BCLC-C liver cancer. They were divided into TACE group $(n=40)$ and combination group ( $\mathrm{n}=35$, TACE plus sorafenib) according to the difference of therapeutic modalities (Figure 1).

\section{Treatment Methods}

Patients in both groups were treated with modified Seldinger method for TACE. The angiographies of celiac, hepatic, inferior phrenic arteries, and superior mesenteric were conducted to identify the feeding arteries of tumor. A 2.8 Fr micro-catheter (Progreat $^{\mathrm{TM}}$, Terumo, Tokyo, Japan) was inserted to feeding arteries of tumor. Oxaliplatin (100-200 mg) and/or fluorouracil glycosides (500-1000 mg) were infused followed by epirubicin (30-60 mg) mixed with 5-25 mL of iodized oil under fluoroscopic monitoring, the dose of chemotherapeutic drugs and super emulsified lipiodol depends on the number, size, and blood supply of tumor lesions.

Patients in the combination group received oral sorafenib (Bayer Pharmaceuticals, 20,131,118, 0.2g/tablet) on the 4th day following TACE treatment. The dose was $400 \mathrm{mg}$, BID. If the intolerable adverse reaction occurred, the dose was adjusted to $200 \mathrm{mg}$, BID, or stop taking Sorafenib after symptom relief for about 2 weeks, usually for at least 3 months or until the disease progresses. During medication, liver disease progressed to Child Pugh Grade C or was lost to follow-up, and drug resistance was discontinued. If new lesions and residual lesions appear at intervals of more than 1 month after re-examination of imaging, both groups can be treated with TACE again.

\section{Observation Indicators}

(1)Efficacy Evaluation After 1 month of treatment, the modified mRECIST standard ${ }^{12}$ was used to evaluate the efficacy. The efficacy evaluation criteria are as follows: Complete Response (CR): disappearance of intra-tumor arterial enhancement in all target lesions; Partial Response (PR): The sum of all the diameters of target lesion size or enhanced lesions at least $30 \%$ is reduced; Stable Disease (SD): the minimum diameter of the target lesion has neither reached the PR nor increased the diameter requirements of the disease progression; Progression Disease (PD): tumor size (The sum of the diameters of all enhanced lesions and the largest diameter of the lesions) increased by at least $20 \%$, or new lesions appeared. (2) Adverse Reactions Refer to the 


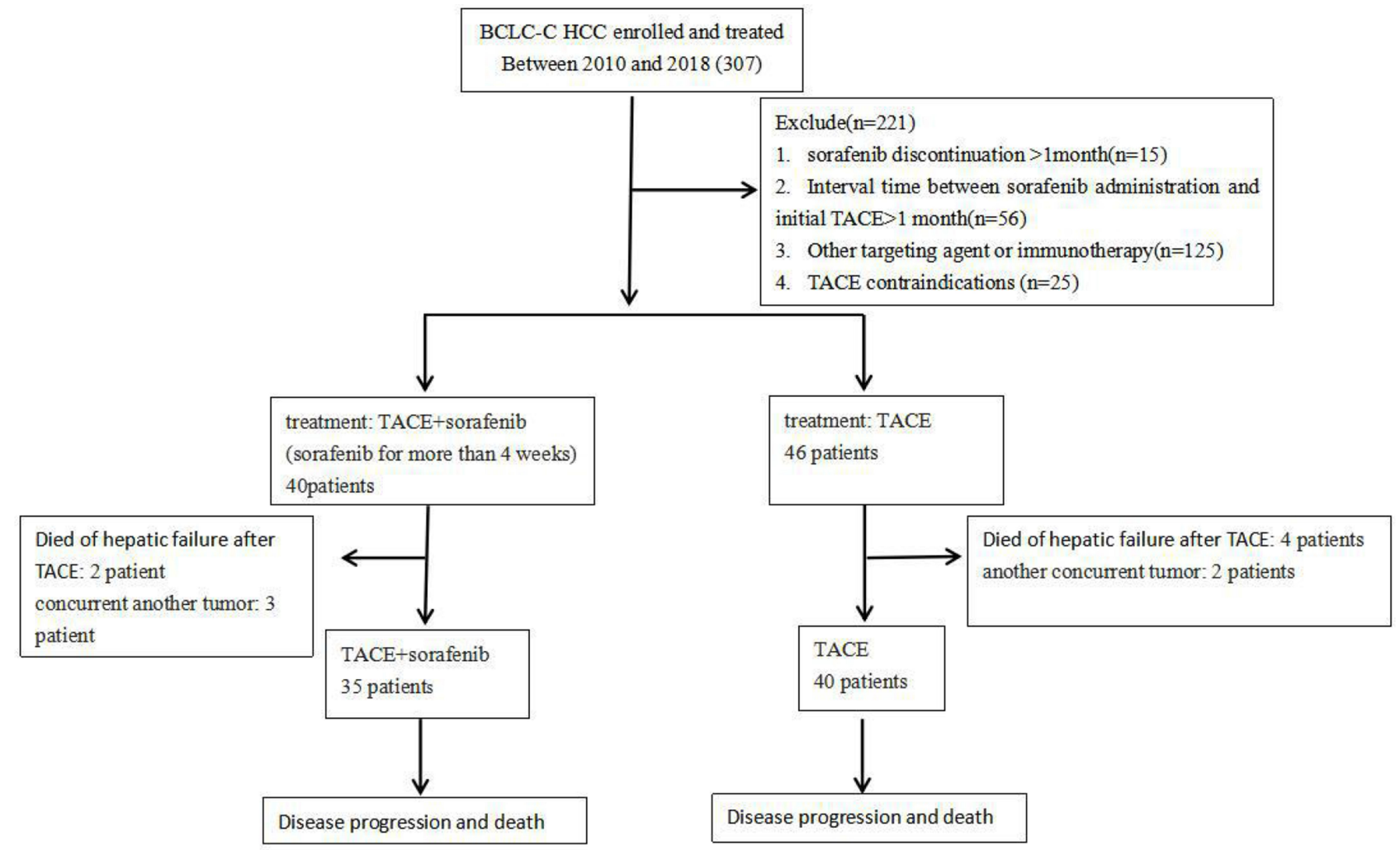

Figure I Flow diagram illustrating the treatment process.

National Cancer Institute's Common Adverse Reaction Rating Standard $3.0^{13}$ to observe the occurrence of adverse reactions in the two groups. (3) Survival Time Calculate the median overall survival (OS) of the two groups. OS is defined as the time from the first TACE after surgery to death or termination of follow-up.

\section{Study Endpoint}

The primary endpoint was overall survival or last follow-up (December 31, 2019). For survival analysis, a follow-up of at least 3 months was considered mandatory. The final date of the follow-up was from an outpatient visit or telephone interview. The secondary endpoint was tumor response as defined by the mRECIST criteria and treatment-related adverse events.

\section{Statistical Methods}

Statistical analysis was performed using SPSS20.0 software. Measurement data were expressed as $\pm \mathrm{s}$, using two independent sample $\mathrm{t}$ tests; count data were taken as examples, using $\chi^{2}$ test; Kaplan-Meier method was used for survival analysis, and comparison was performed by Log rank test. $P<0.05$ was considered statistically significant.

\section{Results}

\section{Comparison of Baseline Data}

There were no significant differences in baseline data $(P>0.05)$ in age, gender, Child-Pugh classification, preoperative AFP, tumor size, number of tumors, history of hepatitis $\mathrm{B}$, and presence or absence of metastasis $(P>0.05)$ (Table 1$)$.

\section{Tumor Response}

After 1 month of treatment, the tumor response was evaluated according to mRECIST standard based on the imaging follow-up results. It was found that the disease control rate of the combined group was significantly better than that of TACE $(\mathrm{P}=0.018)$, suggesting that sorafenib can significantly improve the efficacy of TACE (Table 2).

\section{Adverse Reactions}

The adverse reactions in the two groups were mainly gastrointestinal reactions, bone marrow suppression, skin reactions, and abnormal liver function. In 8 patients, the dose of sorafenib was reduced to $200 \mathrm{mg}$ twice a day due to intolerance. No serious adverse reactions occurred. After symptomatic treatment, the symptoms were alleviated significantly and had no 
Table I Baseline Characteristics of Two Groups' Patients

\begin{tabular}{|c|c|c|c|}
\hline & TACE+Sorafenib(n=35) & $\operatorname{TACE}(n=40)$ & $\mathbf{p}$ \\
\hline Age (years), median (range) & $68.24(35-88)$ & $56(35-85)$ & 0.33 \\
\hline $\operatorname{Sex}(M / F)$ & $30 / 5$ & $32 / 8$ & 0.51 \\
\hline Tumor size $(\mathrm{cm})$, median (range) & $6.9(1.6-12)$ & $7.4(2.1-11.7)$ & 0.28 \\
\hline Tumor number (single/multiple) & $18 / 17$ & $27 / 13$ & 0.15 \\
\hline PVTT(Yes/No) & $24 / 11$ & $28 / 12$ & 0.89 \\
\hline $\mathrm{HBsAg}(\mathrm{Yes} / \mathrm{No})$ & $31 / 4$ & $34 / 6$ & 0.64 \\
\hline $\operatorname{AFP}(\leq 400 \mathrm{ng} / \mathrm{L} />400 \mathrm{ng} / \mathrm{L})$ & $12 / 23$ & $21 / 19$ & 0.11 \\
\hline ECOG $(0-1 />1)$ & $32 / 3$ & $34 / 6$ & 0.39 \\
\hline Child-Pugh class (A/B) & $23 / 12$ & $24 / 16$ & 0.61 \\
\hline Extrahepatic metastasis(Yes/No) & $1 \mathrm{I} / 24$ & $18 / 22$ & 0.23 \\
\hline Number of TACE $(\leq 3 />3)$ & $28 / 7$ & $26 / 14$ & 0.15 \\
\hline Duration of Sorafenib treatment, (days) & 439.4 & - & - \\
\hline
\end{tabular}

Abbreviations: TACE, transarterial chemoembolization; PVTT, portal vein tumor thrombus; HBsAg, hepatitis B surface antigen; AFP, alpha fetoprotein; ECOG, Eastern Cooperative Oncology Group.

Table 3 Treatment-Related AEs in Patients in Two Groups[n(\%)]

\begin{tabular}{|l|l|l|l|l|}
\hline Adverse Events & TACE+Sorafenib(n=35) & TACE(n=40) & $\chi^{\mathbf{2}}$ \\
\hline Nausea & $21(60 \%)$ & $22(55 \%)$ & 0.19 & \\
Diarrhea & $17(48.6 \%)$ & $21(52.5 \%)$ & 0.12 & 0.66 \\
Leukopenia & $20(57 \%)$ & $16(40 \%)$ & 2.19 & 0.73 \\
Fever & $24(69 \%)$ & $28(70 \%)$ & 0.02 & 0.14 \\
Liver function lesion & $6(17.1 \%)$ & $9(22.5 \%)$ & 0.33 & 0.89 \\
Oral Mucositis & $25(71.4 \%)$ & $2(5 \%)$ & 35.75 & 0.56 \\
Fatigue & $24(68.6 \%)$ & $23(57.5 \%)$ & 0.98 & \\
Alopecia & $13(37.1 \%)$ & $10(25 \%)$ & 1.29 & 0.32 \\
Hand-foot skin reaction & $27(77.1 \%)$ & $1(2.5 \%)$ & 44.45 & 10.54 \\
Hypertension & $12(34.3 \%)$ & $2(5 \%)$ & 0.25 \\
\hline
\end{tabular}

Table 2 Summary of Tumor Response[n(\%)]

\begin{tabular}{|l|l|l|l|l|l|l|}
\hline & CR & PR & SD & PD & ORR (\%) & DCR (\%) \\
\hline TACE+Sorafenib & $3(8.57 \%)$ & $12(34.28 \%)$ & $14(40 \%)$ & $6(17.14 \%)$ & $15(42.85 \%)$ & $29(82.86 \%)$ \\
TACE & $2(5 \%)$ & $10(25 \%)$ & $11(27.5 \%)$ & $17(42.5 \%)$ & $12(30.00 \%)$ & $23(57.50 \%)$ \\
$\chi^{2}$ & & & & & 1.34 & 5.64 \\
$P$ & & & & & 0.25 & 0.018 \\
\hline
\end{tabular}

Abbreviations: CR, complete response; PR, partial response; SD, stable disease; PD, progressive disease; ORR, objective response rate; DCR, disease control rate; TACE, transarterial chemoembolization.

effect on subsequent treatment. The incidence of skin reactions, oral mucositis and hypertension in the experimental group was significantly higher than that in the control group $(\mathrm{P}<0.01)$ There was no significant difference in the incidence of nausea and vomiting, abdominal pain and diarrhea, decreased leukocyte, and abnormal liver function in the two groups $(P>0.05)$ (Table 3$)$.

\section{TTP and OS}

Two groups of patients were followed up and found that the half-year, one-year, and two-year survival rates of patients in the TACE group were $57.4 \%, 40.6 \%$, and $21.2 \%$, respectively; the survival rates of patients in the combined group were $83.5 \%, 71.2 \%$, and $45.7 \%$. The overall survival time of patients in the TACE group was 

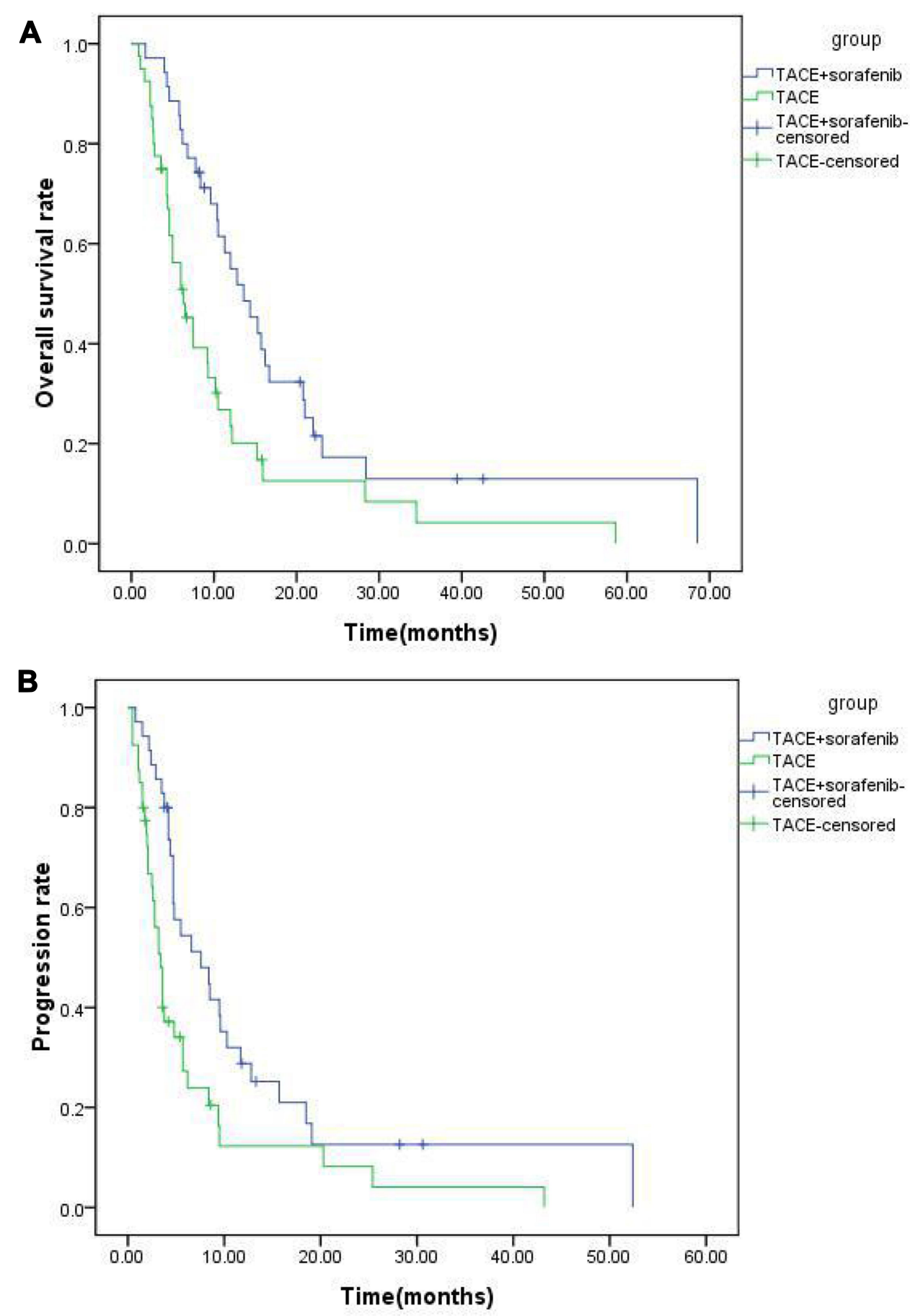

Figure 2 (A) Kaplan-Meier survival curves for comparison of OS between TACE + sorafenib group and TACE group ( $P=0.016)$. (B) Kaplan-Meier survival curves for comparison of TTP between TACE + sorafenib group and TACE group $(P=0.042)$.

6.5 months, and the time to progression was 3.4 months. The total survival time of patients in the combination group was 13.6 months, the time to progression was 7.6 months, and the total survival time of the combination group $(P=0.016$, Figure $2 \mathrm{~A})$ and time to progression $(P=0.042$, Figure $2 \mathrm{~B})$ were significantly better than the TACE group.

\section{Discussion}

TACE plays an important role in the treatment of unresectable advanced HCC. Previous studies have confirmed that TACE can significantly prolong the median survival time of patients with advanced HCC. ${ }^{14,15}$ However, the individual differences among patients receiving TACE are large, and the recurrence 
rate is high. The recurrence rate within 6 months is as high as $29 \% .{ }^{16}$ The curative effect is an important factor restricting its widespread clinical application. Sorafenib, as a commonly used molecular targeted drug, has also shown great application potential in the treatment of advanced HCC. Related studies have confirmed that sorafenib can prolong the overall survival of patients with advanced HCC outstanding drug tolerance. ${ }^{17,18}$ Several international authoritative guidelines recommend sorafenib as the standard treatment for advanced hepatocellular carcinoma, and point out that sorafenib inhibits tumor cell proliferation and inhibits tumor angiogenesis. Early application of sorafenib after palliative surgery may delay tumor recurrence. However, domestic studies mostly focus on patients with BCLC stage B. The overall treatment effect for patients with BCLC stage $\mathrm{C}$ is still unclear. Even for patients with BCLC stage $\mathrm{B}$, there is also some controversy in clinical studies. Domestic studies have shown that TACE combined with sorafenib in the treatment of BCLC Phase B, compared with TACE alone, has not shown significant advantages. ${ }^{19}$ This study explored the overall efficacy of TACE combined with sorafenib in patients with BCLC stage C HCC.

Molecular targeted drugs are the current research hotspots in tumor therapy and one of the new directions in the treatment of hepatocellular carcinoma. Compared with traditional antitumor drugs, it has the advantages of higher pertinence, specificity and less toxic and side effects. The pathogenesis of liver cancer involves numerous signaling pathways. Sorafenib is a small molecule multi-targeted oral multikinase inhibitor. It inhibits Raf/MEK/ERK signaling pathways by inhibiting Raf kinases on the one hand, and inhibits VEGFR-2, VEGFR-3, and PDGFR on the other $-\beta$, FLt-3, c-Kit receptors to inhibit HCC cell proliferation, prevent tumor cell angiogenesis, and effectively induce HCC ectopic planting apoptosis. ${ }^{20,21}$ It is currently the only targeted therapeutic drug that has obtained evidence-based medical evidence that can prolong the survival of hepatocellular carcinoma. Both the SHARP study in Europe $^{22}$ and the Oriental study in Asian populations ${ }^{20}$ have confirmed that patients taking sorafenib have longer disease progression times and overall survival times than patients on supportive care.

Although TACE can treat tumor from both sides of arterial chemotherapy and embolization, TACE also has its shortcomings, mainly because in addition to the main hepatic artery blood supply, liver cancer also receives a portion of the blood supply from the portal vein, which leads to the opening of the hepatic artery-portal communication branch after embolization, leading to incomplete embolism; The effect of TACE on hepatocellular carcinoma with poor blood supply is poor. ${ }^{23}$ In addition, most of the non-necrotic liver cancer tumor cells after TACE are in a proliferative active state. Due to tumor vessel embolism, these tumor cells are in an ischemic and hypoxic state, which can promote some angiogenic factors such as VEGF, serum insulin-like Increased secretion of growth factor-2 (IGF-2), etc., promotes the formation of tumor neovascularization, and the neovascularization and other blood vessels in the liver form a collateral circulation, which also increases the chance of recurrence and metastasis, which affects longterm efficacy. ${ }^{24}$ If an anti-angiogenic drug can be given after liver cancer TACE, it may reduce the chance of recurrence and metastasis in patients with hepatocellular carcinoma, thereby prolonging the survival of patients. TACE and sorafenib targeted therapy theoretically have complementary antitumor effects. Therefore, the combined application of the two in the treatment of primary liver cancer is a hot spot in clinical research. Wei et $\mathrm{al}^{25}$ believe that sorafenib can inhibit tumor cell proliferation and anti-angiogenesis through multi-target inhibition and dual-channel inhibition mechanisms, and complement TACE. Park et al compared the clinical effects of TACE combined with sorafenib and the two alone in treating primary liver cancer. ${ }^{26}$ The results showed that TACE combined with sorafenib had a more significant effect than the two alone.

The data of this study showed that there was no statistically significant difference in the incidence of adverse reactions such as fever, leukopenia, liver damage, and gastrointestinal reactions in patients with TACE combined with sorafenib and those with TACE alone during treatment. There are differences in mucositis, hand-foot-mouth disease, and hypertension, and most of the adverse reactions occur 2 to 4 weeks after medication, and gradually relieve after treatment. This shows that the incidence of adverse reactions in patients with liver cancer treated only by TACE and TACE combined with sorafenib was similar, and the addition of sorafenib did not increase the incidence of adverse reactions. Its adverse reactions mostly occurred 2 to 4 weeks after treatment, and gradually relieved after treatment. This shows that TACE in combination with sorafenib is safe in treating primary liver cancer. Patients with liver cancer were treated with sorafenib after TACE. Not only the short-term efficacy was higher than that of single TACE therapy, but the long-term efficacy was higher than that of TACE group. The median survival at one month, six months, and one year was statistically significant compared with patients in the TACE group, which is consistent with the efficacy of Peng et $\mathrm{al}^{27}$ for the recurrence of postoperative 
recurrence of sorafenib combined with TACE in the treatment of advanced liver cancer.

This study also has certain shortcomings. First, the number of patients enrolled is small, and related cases will continue to be included in subsequent studies for further research. Second, factors that affect the survival time of patients with BCLC stage C are not analyzed, Rather, it simply analyzes the overall disease progression time and survival time, and will further study on the premise that the number of subsequent cases increases.

In summary, patients with BCLC stage $\mathrm{C}$ treated with sorafenib combined with TACE have a gain effect, can significantly benefit patients, and significantly prolong the overall survival time of these patients.

\section{Funding}

This work was supported by the Natural Science Project of Anhui Province of China (No. 1808085MH254).

\section{Disclosure}

The authors report no conflicts of interest for this work.

\section{References}

1. Jemal A, Bray F, Center MM, Ferlay J, Ward E, Forman D. Global cancer statistics. CA Cancer J Clin. 2011;61:69-90. doi:10.3322/caac.20107

2. Bruix J, Sherman M. American Association for the Study of Liver, D. management of hepatocellular carcinoma: an update. Hepatology. 2011;53:1020-1022. doi:10.1002/hep.24199

3. ElSerag HB. Hepatocellular carcinoma. $N$ Engl $J$ Med. 2011;365:1118-1127. doi:10.1056/NEJMra1001683

4. Roayaie S, Jibara G, Tabrizian P, Park JW, Yang J, Yan L. The role of hepatic resection in the treatment of hepatocellular cancer. Hepatology. 2015;62:440-451. doi:10.1002/hep.27745

5. Sheng J, Qin H, Zhang K, Li B, Zhang X. Targeting autophagy in chemotherapy-resistant of hepatocellular carcinoma. Am J Cancer Res. 2018;8:354-365.

6. Marelli L, Stigliano R, Triantos C, et al. Transarterial therapy for hepatocellular carcinoma: which technique is more effective? A systematic review of cohort and randomized studies. Card Inter Rad. 2007;30:6-25. doi:10.1007/s00270-006-0062-3

7. Kudo M, Imanaka K, Chida N, et al. Phase III study of sorafenib after transarterial chemoembolisation in Japanese and Korean patients with unresectable hepatocellular carcinoma. Eur $J$ Cancer. 2011;47:2117-2127. doi:10.1016/j.ejca.2011.05.007

8. Cui HZ, Dai GH, Shi Y, Chen L. Sorafenib combined with TACE in advanced primary hepatocellular carcinoma. Hepatogastroenterology. 2013;60(122):305-310. doi:10.5754/hge12552

9. Wang P, Sheng L, Wang G, et al. Association of transarterial chemoembolization with survival in patients with unresectable hepatocellular carcinoma. Mol Clin Oncol. 2014;2:203-206. doi:10.3892/ mco.2014.239

10. European Association For The Study Of The Liver. European Organisation for research and treatment of cancer. Easleortc clinical practice guidelines: management of hepatocellular carcinoma. J Hepatol. 2012;56:908-943. doi:10.1016/j.jhep.2011.12.001
11. Guglielmi A, Ruzzenente A, Pachera S, et al. Comparison of seven staging systems in cirrhotic patients with hepatocellular carcinoma in a cohort of patients who underwent radiofrequency ablation with complete response. Am J Gastroenterol. 2008;103:597-604. doi:10.1111/j.1572-0241.2007.01604.x

12. Hao H, Zhenhua D, Xiaoran L, et al. Sorafenib combined with transarterial chemoembolization versus transarterial chemoembolization alone for advanced-stage hepatocellular carcinoma: a propensity score matching study. PLoS One. 2014;9:e96620. doi:10.1371/journal.pone. 0096620

13. Trotti A, Colevas AD, Setser A, et al. CTCAE v3.0: development of a comprehensive grading system for the adverse effects of cancer treatment. Semin Radiat Oncol. 2003;13:176-181. doi:10.1016/ S1053-4296(03)00031-6

14. Kong J-Y, Shu-Mei L, Fan H-Y, Zhang L, Zhao H-J, Sheng-Mian L. Transarterial chemoembolization extends long-term survival in patients with unresectable hepatocellular carcinoma. Medicine. 2018;97(33):e11872. doi:10.1097/MD.0000000000011872

15. Wei-Fu L, Liu K-C, Dong L, Zhou C-Z, Cheng D-L, Xiao J-K. Transarterial chemoembolization for hepatocellular carcinoma combined with portal vein tumor vein tumor thrombosis cancer. Manag Res. 2018;10:4719-4726.

16. Yang JD, Aqel BA, Pungpapong S, Gores GJ, Roberts LR, Leise MD. Direct acting antiviral therapy and tumor recurrence after liver transplant for hepatitis C-associated hepatocellular carcinoma. $J$ Hepatol. 2016;65(4)S016882781630318X.

17. Dickinson B, Surawicz CM. Infectious diarrhea: an overview. Curr Gastroenterol Rep. 2014;16:399. doi:10.1007/s11894-014-0399-8

18. Howell J, Pinato DJ, Ramaswami R, et al. On-target sorafenib toxicity predicts improved survival in hepatocellular carcinoma: a multi-centre, prospective study. Aliment Pharmacol Ther. 2017;45:1146-1155. doi:10.1111/apt.13977

19. Wenjin D, Li F, Gong G, Xinming W, Tang T, Jiang X. A comparative study on TACE combined with sorafenib versus TACE in the treatment of BCLC hepatocellular carcinoma. Anti Tumor Pharm. 2015;3:230-234.

20. Cheng AL, Kang YK, Chen Z, et al. Efficacy and safety of sorafenib in patients in the Asia-Pacific region with advanced hepatocellular carcinoma: a phase III randomised, double-blind, placebo-controlled trial. Lancet Oncol. 2009;10:25-34. doi:10.1016/S1470-2045(08)70285-7

21. Cabrera R, Pannu DS, Caridi J, et al. The combination of sorafenib with transarterial chemoembolisation for hepatocellular carcinoma. Aliment Pharmacol Ther. 2011;34:205-213. doi:10.1111/j.13652036.2011.04697.x

22. Llovet JR, Mazzaferro S. Sorafenib improves survival in advanced hepatocellular carcinoma (HCC): Results of a phase III randomized placebo-controlled trial (SHARP trial). J Clin Oncol. 2007;25: LBA1-LBA1.

23. Kang J, Nie Q, Du R, et al. Stereotactic body radiotherapy combined with transarterial chemoembolization for hepatocellular carcinoma with portal vein tumor thrombosis. Mol Clin Oncol. 2014;2:43-50.

24. Pei R. Association of transarterial chemoembolization with survival in patients with unresectable hepatocellular carcinoma. Mol Clin Oncol. 2014;2:203-206.

25. Wei ZG, Lu L, Shao PJ, et al. Observation on the clinical efficacy of TACE combined with sorafenib in the treatment of middle and advanced stage of HCC. Chin J Radiol. 2012;46:252-256. doi:10.1086/665785

26. Park JW, Koh YH, Kim HB, et al. Phase I study of concurrent transarterial chemoembolization and sorafenib in patients with unresectable hepatocellular carcinoma. J Hepatol. 2012;56:1336-1342. doi:10.1016/j.jhep.2012.01.006

27. Peng Z, Chen S, Xiao H, et al. Microvascular invasion as a predictor of response to treatment with sorafenib and transarterial chemoembolization for recurrent intermediate-stage hepatocellular carcinoma. Radiology. 2019;292:237-247. doi:10.1148/radiol.2019181818 


\section{Publish your work in this journal}

Drug Design, Development and Therapy is an international, peerreviewed open-access journal that spans the spectrum of drug design and development through to clinical applications. Clinical outcomes, patient safety, and programs for the development and effective, safe, and sustained use of medicines are a feature of the journal, which has also been accepted for indexing on PubMed Central. The manuscript management system is completely online and includes a very quick and fair peer-review system, which is all easy to use. Visit http://www. dovepress.com/testimonials.php to read real quotes from published authors. 